\title{
A Novel Model for BRT Scheduling Problems
}

\author{
Nguyen Quang Thuan and Phan Nguyen Ba Thang
}

\begin{abstract}
Bus Rapid Transit (BRT) is a public transportation system and plays an important role in urban transportation systems. In order to increase the speed, efficiency, and reliability of BRT systems, the headway optimization and scheduling combination are considered. This work proposes a new multi-objective model for BRT scheduling problems. The problem is then solved by Fast Non-dominated Sorting Genetic Algorithm (NSGA-II) - based method. A case study in Hanoi is investigated. The experimental results show that the new model is applicable.
\end{abstract}

Index Terms-Bus rapid transit, multi-objective, NSGA-II, scheduling.

\section{INTRODUCTION}

Bus Rapid Transit (BRT) can be defined as a bus-based public transport system designed to improve the capacity and reliability of a conventional bus system. A BRT system includes dedicated roadway to buses, and gives priority to buses at intersections. BRT aims to combine the capacity and speed of a metro with the flexibility, lower cost and simplicity of a bus system [1]. BRT is possibly an efficient transport system, especially for cities in developing countries where there are high transit-dependent populations and limited financial resources [2]. Nowadays, the BRT system appears in many cities all over the world such as Bogota, Delhi, Guangzhou, Jakarta, Hanoi, etc.

To improve the efficiency of BRT service or bus systems in general, the headway - the time between two consecutive buses leaving the initial station - is calculated [3], [4]. Another scheduling problem is to plan when BRT vehicles should stop at certain stations. This problem is called the scheduling combination for buses [5], [6]. Sun, Zhou and Wang in 2008 investigated the frequency and scheduling combination for a BRT route in the average time [5]. Most of problems are formulated in the form of an optimization problem [6]-[8]. They are then solved by using heuristic methods [9]-[12].

According to the BRT vehicle operation form and the number of stops, the scheduling is regularly divided into three forms: normal scheduling, zone scheduling and express scheduling. In normal scheduling, vehicles run along the routes and stop at every station from the initial stop to the end.

Manuscript received October 20, 2018; revised June 1, 2019.

Nguyen Quang Thuan is with the International School (VNU-IS), Vietnam National University, Hanoi (VNU), Vietnam (email: nguyenquangthuan@vnu.edu.vn).

Phan Nguyen Ba Thang is with Hanoi University of Science and Technology, Hanoi, Vietnam (email: phanbathang125692@gmail.com).
The vehicle must run at fixed stations and complete the whole routes, as shown in Fig. 1(a). Zone scheduling is defined as vehicles only running on a high-traffic-volume section or zone i.e. vehicles do not stop at some stations (Fig. 1(b)). In express scheduling, vehicles only stop at a certain station with large passenger volume (Fig. 1(c)). In Fig. 1, the black denotes stop and the white does non-stop.

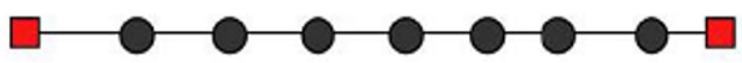

(a)

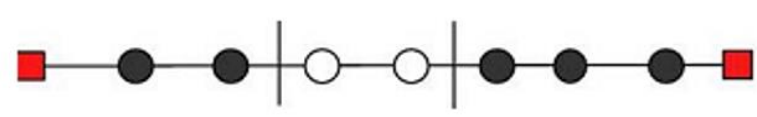

(b)

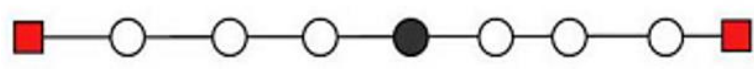

(c)

Fig. 1. (a) Normal schedule; (b) Zone schedule; (c) Express schedule.

It is clear that the design and assignment of BRT vehicles to suitable scheduling forms are transport planners' important tasks. Sun, Zhou and Wang proposed a genetic algorithm (GA) - based method to give the optimal headway and a suitable scheduling combination so that the total cost (including the waiting time, traveling time, operation BRT cost, etc.) is minimized [5]. The authors based on the assumption that a BRT vehicle is not allowed to cross another BRT vehicle. This leads to an unrealistic thing when an express BRT vehicle may cross a normal one.

Our work motivation is to find a new model that cannot only solve the limitation above but also be applicable to real problems. The model in [13] considered the missing passengers because of ambiguity about suitable vehicle list. We will resolve it and in addition, propose an explicit formula for the departure order of vehicle at each stop, which has not appeared before. Moreover, the model also considers using bus performance of the operators. The model is in the form of a multi-objective optimization problem with fewer variables, constraints and more clarity. The proposed model is solvable since we can adapt NSGA-II [9], [14], an efficient heuristic method for solving multi-objective optimization problems.

To investigate how the model works, we consider a case study in Hanoi that is for Yen Nghia - Kim Ma BRT. The promising experimental results make a valuable contribution of this work.

The structure of the paper is organized as follows. After the introduction, Section II describes BRT systems and the mathematical problem. The NSGA-II applied to the problem is presented in Section III. Section IV investigates the experimental results before the conclusion in Section V. 


\section{FORMULATION}

\section{A. Assumptions}

The problem is modeled under some assumptions:

i) BRT vehicles are completely prioritized, i.e. they never stop due to traffic lights;

ii) BRT vehicles run at constant speed, namely, the running time between two any stations is not changed;

iii) The headway is fixed in the study period;

iv) The passenger arrival rate is uniform and unchanged in the given period;

v) The duration for stop and acceleration and deceleration are fixed.

\section{B. Notations}

All parameters in the model:

$M$ - the total number of operating vehicles in the study period,

$N$ - the total number of stops on the route,

$T$ - studied period,

$T_{0}$ - dwelling duration at every stop,

$c$ - acceleration and deceleration duration,

$h$ - headway,

$L$ - the optimal number of on-board passengers,

$t_{j}$ - the running time of vehicles between stop $j-1$ and $j$,

$r_{j, k}$ - the arrival rate of passengers at stop $j$ wanting to go to stop $k(k>j)$.

All variables in the model:

$d_{j}^{i}$ - the departure time vehicle $i$ at stop $j$,

$l_{j, k}$ - total vehicles stop at both $j$ and $k$,

$\delta_{j}^{i}$ - binary variable, gets 1 if vehicle $i$ stops at $j$, otherwise is 0 ,

$\delta_{j, k}^{i}$ - binary variable, gets 1 if vehicle $i$ stops at $j$ and $k$, otherwise is 0 ,

$(I, j, k)$ - an integer and gets the value $i$ if vehicle $i$ actually leaving stop $j$ in $I$ th place of all vehicle stop at both $j$ and $k$.

$T_{j, k}^{i}$ - duration time of vehicle $i$ moving from $j$ to $k$,

$A_{j}^{i}$ - the number of alighting passengers at stop $j$ from vehicle $i$,

$B_{j}^{i}$ - the number of boarding passengers at stop $j$ from vehicle $i$,

$L_{j}^{i}$ - the number of passengers on vehicle when vehicle $i$ runs from $j$ to $j+1$.

\section{Formulation}

\section{Objective function}

The first objective function is written as follows

$$
\begin{aligned}
f_{1}= & \sum_{j=1}^{N} \sum_{k=j+1}^{N} \sum_{I=1}^{l_{j, k}} r_{j, k} \cdot \frac{\left(d_{j}^{(I, j, k)}-d_{j}^{(I-1, j, k)}\right)^{2}}{2} \\
& +\sum_{j=1}^{N} \sum_{k=j+1}^{N} \sum_{I=1}^{l_{j, k}} r_{j, k} \cdot\left(d_{j}^{(I, j, k)}-d_{j}^{(I-1, j, k)}\right) \cdot T_{j}^{(I, j, k)}
\end{aligned}
$$

where the first term is the average passengers waiting time for vehicle Ith at stop $j$ in the duration of $\left(d_{j}^{(I, j, k)}-d_{j}^{(I-1, j, k)}\right)$ and the second term is the total travel time on-board of $r_{j, k} .\left(d_{j}^{(I, j, k)}-d_{j}^{(I-1, j, k)}\right)$ passengers from $j$ to $k$ for vehicle $I$ th.

$$
f_{2}=\sum_{i=1}^{M} \sum_{j=1}^{N}\left|L_{j}^{i}-L\right| \cdot\left(d_{j+1}^{i}-d_{j}^{i}\right)
$$

Each vehicle has a certain capacity of $L$ seats and $L_{j}^{i}$ is the number of passengers on vehicle $i$ travelling from $j$ to $j+1$. Therefore, the value $\left|L_{j}^{i}-L\right| .\left(d_{j+1}^{i}-d_{j}^{i}\right)$ will measure the efficiency of using vehicle $i$ on the link $(j, j+1)$. Thus, the second function expresses the efficiency or performance of vehicle operators.

The problem is modeled by the form $V_{\min }\left\{f_{1}, f_{2}\right\}$ i.e. it is a bi-objective optimization problem.

\section{Time constraints}

The departure time at stop 1 for every vehicle:

$$
d_{1}^{i}=(i-1) \cdot h, \quad i=\overline{1, M}
$$

The departure time of vehicle $i$ at stop $j$ is equal to the sum of the departure time of that vehicle at stop $j-1$ and the running time and acceleration/deceleration time and the dwelling time:

$$
d_{j}^{i}=d_{j-1}^{i}+t_{j}+\left(\delta_{j-1}^{i}+\delta_{j}^{i}\right) \cdot c+\delta_{j}^{i} \cdot T_{0}
$$

The binary variable $\delta_{j}^{i}$ determines whether vehicle $i$ stops at $j$ or not. Hence, the following binary variable indicates whether vehicle $i$ stops at both $j$ and $k$ or not:

$$
\delta_{j, k}^{i}=\delta_{j}^{i} \cdot \delta_{k}^{i}
$$

Given $M$ vehicles, the total number of vehicles stopping at both $j$ and $k$ is calculated:

$$
l_{j, k}=\sum_{i=1}^{M} \delta_{j, k}^{i}
$$

Since the situation of crossing among vehicles, the order of BRT vehicles leaving the station $j \neq 1$ is probably not the same. And BRT vehicle $i$ can leave stop $j$ in $I$ th place of all vehicle stop at both $j$ and $k($ then $(I, j, k)=i)$, with:

$$
I=1+\delta_{j, k}^{i} \cdot \sum_{m=1, m \neq i}^{M} \delta_{j, k}^{m} \cdot f(i, j, m)
$$

$$
f(i, j, m)= \begin{cases}1 & \left(d_{j}^{i}>d_{j}^{m}\right) \text { and }\left(\left(d_{j}^{i}=d_{j}^{m}\right) \text { or }(i>m)\right) \\ 0 & \left(d_{j}^{i}<d_{j}^{m}\right) \text { and }\left(\left(d_{j}^{i}=d_{j}^{m}\right) \text { or }(i<m)\right)\end{cases}
$$

In equation (6), $f(i, j, m)=1$ means that vehicle $i$ leaves stop $j$ after vehicle $m$. This happens in two cases. In the first case, at stop $j$, the departure time of vehicle $i$ is greater than the one of vehicle $m\left(d_{j}^{i}>d_{j}^{m}\right)$. In the second case, vehicle $i$ 
and vehicle $m$ have the same departure time but vehicle $i$ leaves stop 1 after vehicle $m\left(d_{j}^{i}=d_{j}^{m}\right)$ and $(i>m)$. The value $\delta_{j, k}^{m}$ in equation (5) tells that only vehicle $m$ which stops at both $j$ and $k$ is considered. $\sum_{m=1, m \neq i}^{M} \delta_{j, k}^{m} . f(i, j, m)$ in equation (5) calculates the number of vehicles leaving stop $j$ before vehicle $i$, among all of vehicles stopping at both $j$ and $k$. Therefore, the departure order of vehicle $i$ is expressed in equation (5).

The moving time of vehicle $I$ th from $j$ to $k$ :

$$
T_{j, k}^{(I, j, k)}=d_{k}^{(I, j, k)}-d_{j}^{(I, j, k)}-T_{0}
$$

\section{Number passenger constraints}

The number of alighting passengers in vehicle $i$ at stop $j$ equals the sum of boarding passenger number at all stops before $j$ :

$$
A_{j}^{i}=\delta_{j}^{i} \cdot \sum_{k=1}^{j-1} \delta_{k}^{i} \cdot r_{k, j} \cdot\left(d_{k}^{(I, k, j)}-d_{k}^{(I-1, k, j)}\right)
$$

The number of boarding passengers in vehicle $i$ at stop $j$ equals the sum of alighting passenger number at all stops after j:

$$
B_{j}^{i}=\delta_{j}^{i} \cdot \sum_{k=j+1}^{N} \delta_{k}^{i} \cdot r_{j, k} \cdot\left(d_{j}^{(I, j, k)}-d_{j}^{(I-1, j, k)}\right)
$$

Number of on-board passengers of vehicle $i$ from stop $j$ to stop $j+1$ equals the one from the stop $j-1$ to $j$ plus boarding passengers at stop $j$ minus alighting passengers at stop $j$ :

$$
L_{j}^{i}=L_{j-1}^{i}+B_{j}^{i}-A_{j}^{i}
$$

In summary, the scheduling combination problem can be formulated as follows: $V_{\min }\left\{f_{1}, f_{2}\right\}$, subject to the constraints (1)-(10). Compare with the existed models, the proposed model has fewer constraints and variables. However, it is still 0-1 integer non-linear two-objective programming problem that is a hard problem to determine the exact solution. The following section shows a heuristic approach for the solution method.

\section{SOLUTION BASED METHOD - NSGA II}

\section{A. Introduction to NSGA - II}

In a multi-objective optimization problem, a feasible solution $x^{l}$ is said to dominate another solution $x^{2}$ if both the following conditions are true: (i) The solution $x^{l}$ is no worse than $x^{2}$ in all objectives; and (ii) The solution $x^{l}$ is strictly better than $x^{2}$ in at least one objective. The set of Pareto optimal solutions are not dominated by any other feasible solutions. In Fig. 2, solutions that lie along the front are non-dominated solutions while those that lie inside the line are dominated because there is always another solution on the line that has at least one objective that is better. Solving a multi-objective optimization problem is to find the set of Pareto optimal solutions.

When a multi-objective problem is linear, i.e. all the objective functions and constraint functions are linear, there exists efficient solution methods [15], [16]. It is hard to solve a multi-objective problem if the non-linearity and discrete variables appear. In such a difficult case, a genetic algorithm could be a solution method.

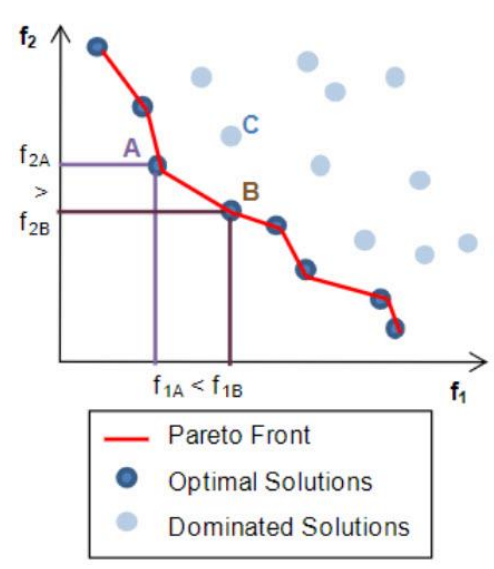

Fig. 2. Example of a Pareto front [17].

Genetic algorithm (GA) is a branch of evolutionary computation in which one imitates the biological processes of reproduction and natural selection to solve for the 'fittest' solutions. GA allows one to find solutions to problems that other optimization methods cannot handle due to a lack of continuity, derivatives, linearity, or other features. Fast non-dominated sorting genetic algorithm (NSGA-II, see [9], [14] for more detail) is a multi-objective evolutionary algorithm (MOEAs). NSGA-II used a fast non-dominated sorting approach with $\mathrm{O}\left(\mathrm{MN}^{2}\right)$ (where $\mathrm{M}$ is the number of objectives and $\mathrm{N}$ is the population size). In most problems, NSGA-II is able to find a good Pareto front and convergence near the true Pareto-optimal front.

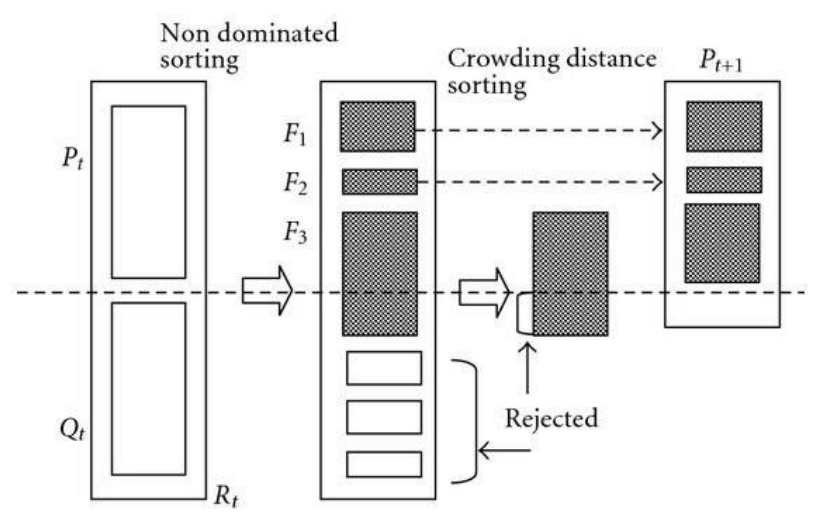

Fig. 3. NSGA II scheme.

The general scheme of NSGA-II is shown in Fig. 3. NSGA-II has two basic procedures:

i) Fast non-dominated sorting:

The procedure will separate all of solutions into many fronts. All solutions in the $i$ th front will dominate all solutions in the $j$ th front $(i<j)$, i.e. all of the objective values of solutions in $i$ th front are better than all solutions in the front inside it.

ii) Crowding distance assignment: 
Crowding distance of a solution in a front is average distance of two points in either side of this point along each of the objectives. The smaller crowding distance is, the higher density level around a solution is. In a front, a solution will be selected for the next generation if it has enough small crowding. Crowding distance is the basis for the selection process. Of course, if a solution is selected, all solutions in outside fronts will be also selected.

Pseudocode for NSGA-II:

procedure NSGA-II $\left(N, N_{A}\right)$

$$
\begin{aligned}
& t \leftarrow 0 \\
& P_{t} \leftarrow \text { new population }(N) \\
& Q_{t} \leftarrow \emptyset \\
& A \leftarrow \text { non dominated }\left(P_{t}\right) \\
& \text { while not stop criterion do } \\
& R_{t} \leftarrow P_{t} \cup Q_{t} \\
& F \leftarrow \text { fast non dominated sorting }\left(R_{t}\right) \\
& P_{t+1} \leftarrow \varnothing \\
& i \leftarrow 1 \\
& \text { while }\left|P_{t+1}\right|+\left|F_{i}\right| \leq N \text { do } \\
& \quad C_{i} \leftarrow \operatorname{crowding} \text { distance assignment }\left(\mathrm{Fi}_{\mathrm{i}}\right) \\
& P_{t+1} \leftarrow P_{t} \cup F_{i} \\
& i \leftarrow i+1 \\
& \text { end while } \\
& F_{i} \leftarrow \operatorname{sort}\left(F_{i}, C_{i},\right. \text { 'descending') } \\
& P_{t+1} \leftarrow P_{t+1} \cup F_{i}\left[1:\left(N-\left|P_{t+1}\right|\right)\right] \text { (fill } P_{t+1} \\
& \text { with the } \left.N-\left|P_{t+1}\right| \text { less crowded individuals of } F_{i}\right) \\
& Q_{t+1} \leftarrow \operatorname{selection}\left(P_{t+1}, N\right) \\
& Q_{t+1} \leftarrow \operatorname{crossover}\left(Q_{t+1}\right) \\
& Q_{t+1} \leftarrow \operatorname{mutation}\left(Q_{t+1}\right) \\
& t \leftarrow t+1 \\
& A \leftarrow \text { non dominated }\left(A \cup Q_{t}\right) \\
& \text { end while } \\
& \text { end procedure }
\end{aligned}
$$

In this algorithm:

- $\mathbf{P} \leftarrow$ new population( $\mathbf{N})$ generates a random population with $\mathbf{N}$ individuals following the encoding scheme which has been adopted for the problem,

- $\mathbf{A} \leftarrow$ non dominated(P) returns the individuals which lie in the first front of the population $\mathbf{P}$,

- $\mathbf{F} \leftarrow$ fast non dominated sorting(P) employs fast non-dominated sorting procedure to find the front of each solution in population $\mathbf{P}$,

- $\mathbf{C}_{\mathbf{i}} \leftarrow$ crowding distance assignment $\left(F_{\mathbf{i}}\right)$ employs crowding distance assignment procedure to estimate how the solutions of front $i$ are spread in the objective space.

\section{B. Applying NSGA - II to the problem}

In our problem, each one of three scheduling forms is easily encoded by using a 2-bit binary number: 01 represents the normal scheduling, 10 is the zone scheduling and 11 is assigned to the express scheduling. The length of chromosomes depends on the frequency or the headway. For example, consider the study period $T=15$ minutes with the headway $h=3$ minutes. That means the number of vehicles operated $M=5$. If an individual has the chromosome of 1110 110110 then it is understood as that 5 vehicles are assigned to $11,10,11,01$ and 10 scheduling form, respectively. As well as other genetic algorithms, NSGA-II has some operators:

i) Selection:

Given a population at a certain generation, Selection determines which individuals are chosen for the next generation based on crowding distance and fronts.

ii) Crossover:

Two individuals cross at a random position. For example, in Fig. 4, the crossing of three head genes of 0101101011 and two tail genes of 1110010110 is 0110100110 and 11 10011011 . The crossing rate is set to $P_{c}$. It means that after selecting a random crossover position, one chooses a random value in the range from 0 to 1 . If the random value is less than or equal to $P_{c}$, the crossover process will succeed and vice versa, if the random value is greater than $P_{c}$, the process will fail.

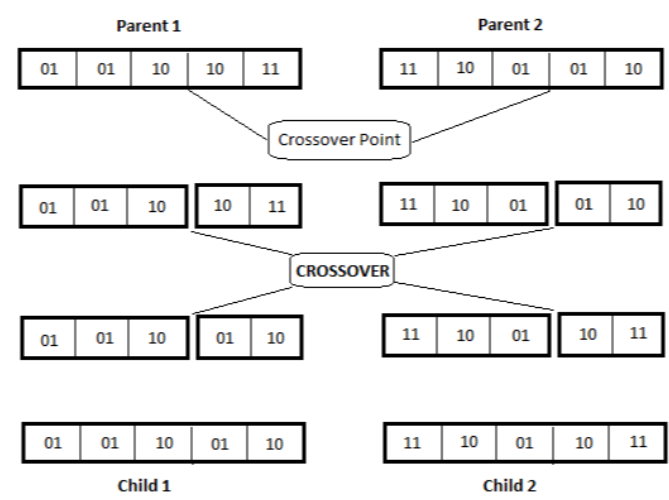

Fig. 4. Crossover process.

iii) Mutation:

Given a mutation rate of $\mathrm{P}_{\mathrm{m}}$. The individual is mutated at a random position, namely:

- "01" becomes "10" or "11",

- "10" becomes "01" or "11",

- "11" becomes "01" or "10".

\section{EXPERIMENT RESULTS}

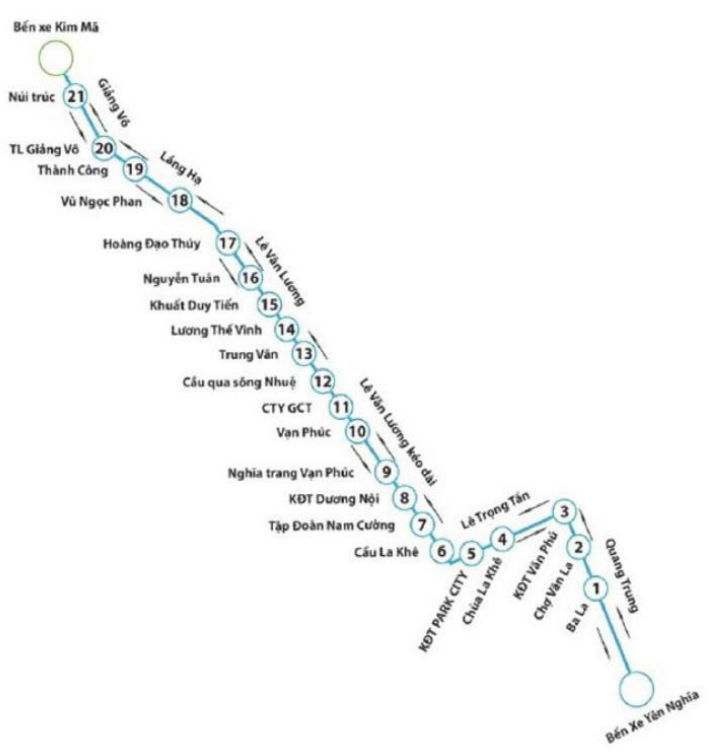

Fig. 5. Kim Ma- Yen Nghia BRT route.

Hanoi, the capital of Vietnam, is planning to have 8 BRT 
routes by 2030. At present, Kim Ma - Yen Nghia BRT route (Fig. 5) has been completed and used since the end of 2017. We apply the model to this route.

The BRT route is investigated with the following parameters:

The study period is $T=1 \mathrm{~h}$ and the number of stops is $N=23$, $T_{0}=30 \mathrm{~s}$ and $c=6 \mathrm{~s}$.

The average speed is $45 \mathrm{~km} / \mathrm{h}=750 \mathrm{~m} / \mathrm{min}$. Based on the distance and the speed, the running time is determined in Table I:

TABLE I: RUNNING TIME ON LINK

\begin{tabular}{|c|c|c|}
\hline From - To & Distance & Time \\
\hline Yen Nghia-1 & 1589 & 2.12 \\
\hline $1-2$ & 511 & 0.68 \\
\hline $2-3$ & 512 & 0.68 \\
\hline $3-4$ & 548 & 0.73 \\
\hline $4-5$ & 503 & 0.67 \\
\hline $5-6$ & 417 & 0.55 \\
\hline $6-7$ & 470 & 0.63 \\
\hline $7-8$ & 630 & 0.84 \\
\hline $8-9$ & 550 & 0.73 \\
\hline $9-10$ & 410 & 0.55 \\
\hline $10-11$ & 590 & 0.79 \\
\hline $11-12$ & 585 & 0.78 \\
\hline $12-13$ & 565 & 0.75 \\
\hline $13-14$ & 720 & 0.96 \\
\hline $14-15$ & 640 & 0.85 \\
\hline $15-16$ & 625 & 0.84 \\
\hline $16-17$ & 1130 & 1.50 \\
\hline $17-18$ & 665 & 0.89 \\
\hline $18-19$ & 840 & 1.12 \\
\hline $19-20$ & 720 & 0.96 \\
\hline $20-21$ & 770 & 1.02 \\
\hline $21-$ Kim Ma & 710 & 0.95 \\
\hline
\end{tabular}

TABLE II: THREE SCHEDULING FORMS

\begin{tabular}{|c|c|c|}
\hline Normal Scheduling & Zone Scheduling & Express Scheduling \\
\hline 1 & 1 & 1 \\
\hline 1 & 0 & 0 \\
\hline 1 & 0 & 0 \\
\hline 1 & 1 & 1 \\
\hline 1 & 1 & 1 \\
\hline 1 & 1 & 0 \\
\hline 1 & 0 & 0 \\
\hline 1 & 0 & 0 \\
\hline 1 & 0 & 0 \\
\hline 1 & 1 & 0 \\
\hline 1 & 1 & 1 \\
\hline 1 & 1 & 0 \\
\hline 1 & 0 & 0 \\
\hline 1 & 1 & 0 \\
\hline 1 & 1 & 1 \\
\hline 1 & 0 & 0 \\
\hline 1 & 0 & 0 \\
\hline 1 & 1 & 1 \\
\hline 1 & 1 & 0 \\
\hline 1 & 0 & 0 \\
\hline 1 & 0 & 0 \\
\hline 1 & 0 & 0 \\
\hline 1 & 1 & 1 \\
\hline
\end{tabular}

Based on the passenger arrival rates, three scheduling forms are chosen as below in Table II. For the normal scheduling, vehicles catch/take passengers at every station; For the zone scheduling, they stop at $12 / 23$ and $7 / 23$ for the express scheduling. In the table below, " 1 " means stop and " 0 " is non-stop.

The algorithm was coded by Python and on a computer that has the configurations: Core i5, RAM 8GB, CPU $2.49 \mathrm{GHz}$. The NSGA II runs with parameters: the number of generations is 500 and the size of populations is 150 . The algorithm is implemented five times with five different numbers of BRT vehicles $M=8,10,12,15$ and 20 . In the study period $T=1 h$, the corresponding headway $h$ (60 $\mathrm{min} /$ number of vehicles) is $7.5,6.0,5.0,4.0,3.0$, respectively.

The running time is quite small. It is only in seconds. The result is reported in Fig. 6. The horizontal axis shows the total passengers' cost while the vertical axis expresses the efficiency or performance of vehicle operators. Five lines are presented in five colors: yellow, black, red, green and blue. Each line is an approximation of Pareto front corresponding to a value of headway $h$.

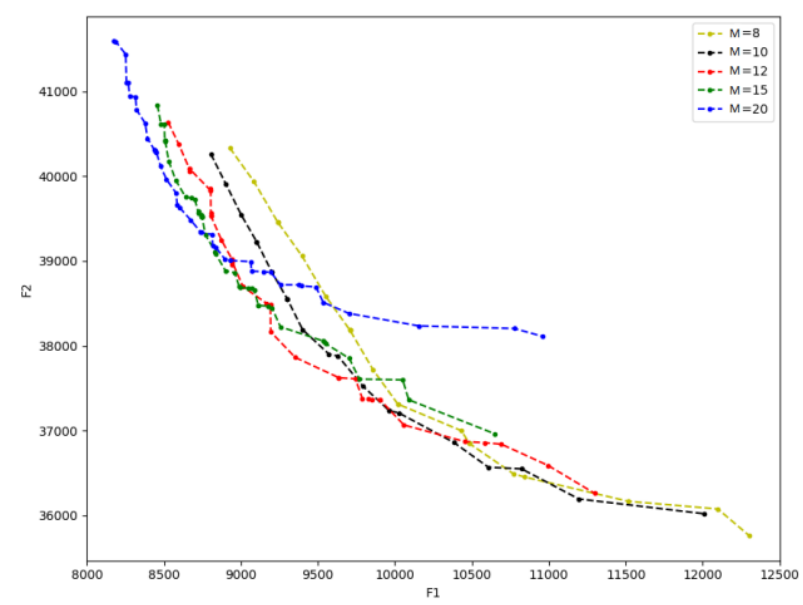

Fig. 6. NSGA-II result.

Some remarks on the result:

In a front, when the number of zones and express vehicles is increased, the total cost of the average passengers waiting time and the travel time on-board of passengers is decreasing and the total cost of performance of vehicle operator is increasing.

The best-known front is formed from 5 obtained good fronts. Based on a suitable ratio of the total passengers' cost and the total performance of operators, decision makers could select a rational solution from this front. For instance, if $f_{1}$ is around 8500 and $f_{2}$ is approximately 40,000, a solution is chosen from the blue front representing the number of vehicles $M=20(h=3)$; or if $f_{1}$ is around 9500 and $f_{2}$ is approximately 37,500 , a reasonable solution is from the red front representing the number of vehicles $M=12(h=5)$.

\section{CONCLUSION}

The paper proposed a new multi-objective model for BRT scheduling problems. It overcomes existing models in some senses such as the explicit formula for the departure order of vehicles at each stop; the consideration to the performance of vehicle operators; the fewer variables and constraints.

The NSGA-II method is adapted for solving the model. The case study of Yen Nghia - Kim Ma BRT route is investigated. The quickly obtained results efficiently support decision makers to schedule a BRT route.

In the future, the model can be considered in the case of integrating traffic lights. At this point, traffic lights could be 
considered as virtual stops where the number of passengers boarding/alighting is zero.

\section{REFERENCES}

[1] H. Levinson, S. Zimmerman, J. Clinger et al., Case Studies in Bus Rapid Transit, TCRP Report 90, vol. 1, Transportation Research Board, Washington, USA, 2003.

[2] T. Rickert, Technical and Operational Challenges to Inclusive Bus Rapid Transit: A Guide for Practitioners, World Bank, Washington, USA, 2010.

[3] Z. J. Bai, G. G. He, and S. Z. Zhao, "Design and implementation of Tabu search algorithm for optimizing BRT Vehicles dispatch," Computer Engineering and Application, vol. 43, no. 23, pp. 229-232, 2007.

[4] S. Liang, Z. He, and Z. Sha, "Bus rapid transit scheduling optimal model based on genetic algorithm," in Proc. ICCTP 2011, 2011, pp. 1296-1305.

[5] C. Sun, W. Zhou, and Y. Wang, "Scheduling combination and headway optimization of bus rapid transit," Journal of Transportation Systems Engineering and Information Technology, vol. 8, no. 5, pp. 61-67, 2008.

[6] C. Avishai, "Urban transit scheduling: Framework, review and examples," Journal of Urban Planning and Development, vol. 128, no. 4, pp. 225-243, 2002.

[7] L. G. Dai and Z. D. Liu, "Research on the multi-objective assembled optimal model of departing interval on bus dispatch," Journal of Transportation Systems Engineering and Information Technology, vol. 7, no. 4, pp. 43-46, 2007.

[8] M. A. Miller, Y. Yin, T. Balvanyos, and C. Avishai, "Framework for bus rapid transit development and deployment planning," Research report, California PATH, University of California Berkeley, 2004.

[9] K. Deb, A. Pratap, S. Agarwal, and T. Meyarivan, "A fast and elitist multi-objective genetic algorithm," IEEE Transactions on Evolutionary Computation, vol. 6, no. 2, pp. 182-197, 2002.

[10] Q. S. Fan and W. Pan, "Application research of genetic algorithm in intelligent transport systems scheduling of vehicle," Computer and Digital Engineering, vol. 35, no. 5, pp. 34-35, 2007.

[11] P. Shrivastava and S. L. Dhingra, "Development of coordinated schedules using genetic algorithms," Journal of Transportation Engineering, vol. 128, no. 1), pp. 89-96, 2002.

[12] G. Tong, "Application study of genetic algorithm on bus scheduling," Computer Engineering, vol. 31, no. 13, pp. 29-31, 2005.

[13] Q. T. Nguyen and N. B. T. Phan, "Scheduling problem for bus rapid transit routes," Advances in Intelligent Systems and Computing, vol. 358(B), pp. 69-79, 2015.

[14] R. Schaefer, Foundations of Global Genetic Optimization, Studies in Computational Intelligence, vol. 74, Springer, 2007.
[15] H. Benson, "An outer approximation algorithm for generating all efficient extreme points in the outcome set of a multiple objective linear programming problem," Journal of Global Optimization, vol. 13, pp. 1-24, 1998.

[16] B. L. Gorissen and D. D. Hertog, "Approximating the pareto set of multi-objective linear programs via robust optimization," Operations Research Letters, vol. 4, no. 5, pp. 319-324, 2012.

[17] C. Villa and R. Labayrade, "Energy efficiency vs subjective comfort: A multi-objective optimization method under uncertainty," in Proc. 12th Conference of International Building Performance Simulation Association, Sydney, 14-16 November, 2011, pp. 1905-1912.

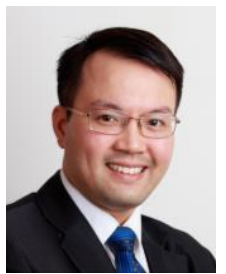

Nguyen Quang Thuan was graduated from Hanoi University of Science and Technology in 2004 with the engineer diploma of applied mathematics and informatics. He got the master and $\mathrm{PhD}$ degree in informatics from University of Paul Verlaine Metz, France in 2007 and 2010 respectively. Right after having $\mathrm{PhD}$ degree, he spent one-year postdoc at Technical University of Freiberg, Germany.

He was a lecturer at School of Applied Mathematics and Informatics, Hanoi University of Science and Technology (HUST) from 2004 to 2017. He was the head of the Department of Applied Mathematics from 2014. Being a vice-dean of International School (VNU-IS), he has worked for Vietnam National University, Hanoi (VNU) since 2017.

Dr. Nguyen is a member of the Vietnam Mathematics Society. His research interest focuses on solving large-scale optimization problems in different fields such as transportation, traffic, communication, manufacturing, etc.

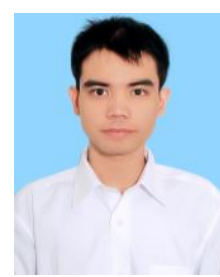

Phan Nguyen Ba Thang was born in Bac Thanh, Yen Thanh, Nghe An in 1992. He was graduated from Mathematics and Informatics Engineer of Hano University of Science and Technology in 2015.

He works for Nippon Steel \& Sumikin Metal Products Vietnam (NSMV) since 2017 as an artificial intelligence engineer. $\mathrm{He}$ has some publications: Scheduling problem for bus rapid transit routes, Advances in Intelligent Systems and Computing, Springer, pp. 69-79, vol 358 (ICCSAMA-2015); A New approach for optimizing traffic signals in networks considering rerouting, Advances in Intelligent Systems and Computing, Springer, pp. 143-154, vol. 359 (MCO-2015). His current researches are in image processing, machine learning, deep learning in computer vision.

Mr. Phan has won Hoa Trang Nguyen Awards and First Prize in the Student's Scientific Research Contest 2015. 